\title{
Metódica de la biopsia intestinal. Experiencia en 140 casos
}

Dr. Simón Lam Rojas*

\begin{abstract}
A successtull technigue is desc ribed of intesinal peroral biopsy with the minimum une of instrumental inplements. T'lis was practiced on 140 chidren whuse ages Ductuated from 1 w 13 yeare oild.

Intravenous Diazepatn assuciated to oral Meliclerpramida was uged as pre medication. The sedation was cunsidered salistactury in $90 \%$ of 1 he cases.

Intestinal biopsy was pertormed when spontaneous bile drainage at $\mathrm{pH}$ t occurred. It was obtained on 9260 of the cases. This tact made the radiulogical control innecessary.

In $42 \%$ of the cases udequale samples of intestinal mucosa were obtaned for hystological investikation. These results ure comparable to others using more complex methods.

Vone secondary complications were registered while applying both the premedisation und the livpsy prucedure.
\end{abstract}

La incorporación de la técnica de la biopsia intestinal peroral, hace aproximadamente dos décadas, ha contribuido notablemente al estudio, diagnóstico y contrul evolutivo de las afecciones enterales, dia-

* Servicio de Pediatria. Unidad de Gastrunenterulogía. Haspital Kegional de Tulea. rrea aguda y crónica, síndromes de malabsorción, y de la Enfermedad Celíaca, en particular.(1, 2)

La biopsia intestinal peroral es un procedimiento que proporciona importantes datos relativos a las alteraciones de las funciones intestinales a través del estudio histológico, bioquímico e inmunológico de la mucosa intestinal. ${ }^{(3)}$ 
La Enfermedad Celíaca es una entidad clínica relativamente frecuente en nuestro país, su pilar diagnóstico está constituido por el estudio histológico de la mucosa intestínal. ${ }^{.4,5}$

La incidencia real de la enfermedad se desconoce, fundamentalmente, porque id biopsia intestinal se encuentra limitada a algunos centros especializados, dado que requiere instrumental adecuado, personal adiestrado, servicio de radiología y, finalmente, posibilidad de estudio histopatologico!s. on

For otra parte, como la Enfermedad Celíaca conlleva una intolerancia permanente al gluten y, como se ha demostrado, puede complicarse de linfurna, existe el consensir de que el paciente portador de este trastorno deba ser privado indelinidamente de esta proteína. Como consecuencia, es imperativo practicar la biopsia intestinal peroral como nedio diagnóstico insustituible en todo paciente que presente síntomas compatibles con dicha enfermedad. ${ }^{\text {5. } i) ~}$

Al respecto, es nutable la escasez de publicaciones extranjeras y nacionales que se refieren a la metordica de la biopsia intestinal, lo que limita al neófito en el interćs para practicar esta técnica.

La biopsia inte: tinal perural es un procedimiento relativamente simple, que requiece una mínima implementación instrumental y debidamente controlada puede efectuarse sin apuyo radiológico. La muestra de mucosa obtenida, una vez fijada, puede enviarse a cualquier centro que cuente con un patologo entrenado y aportara valiosisimas informaciones para el clínico.

\section{FOTO 1}

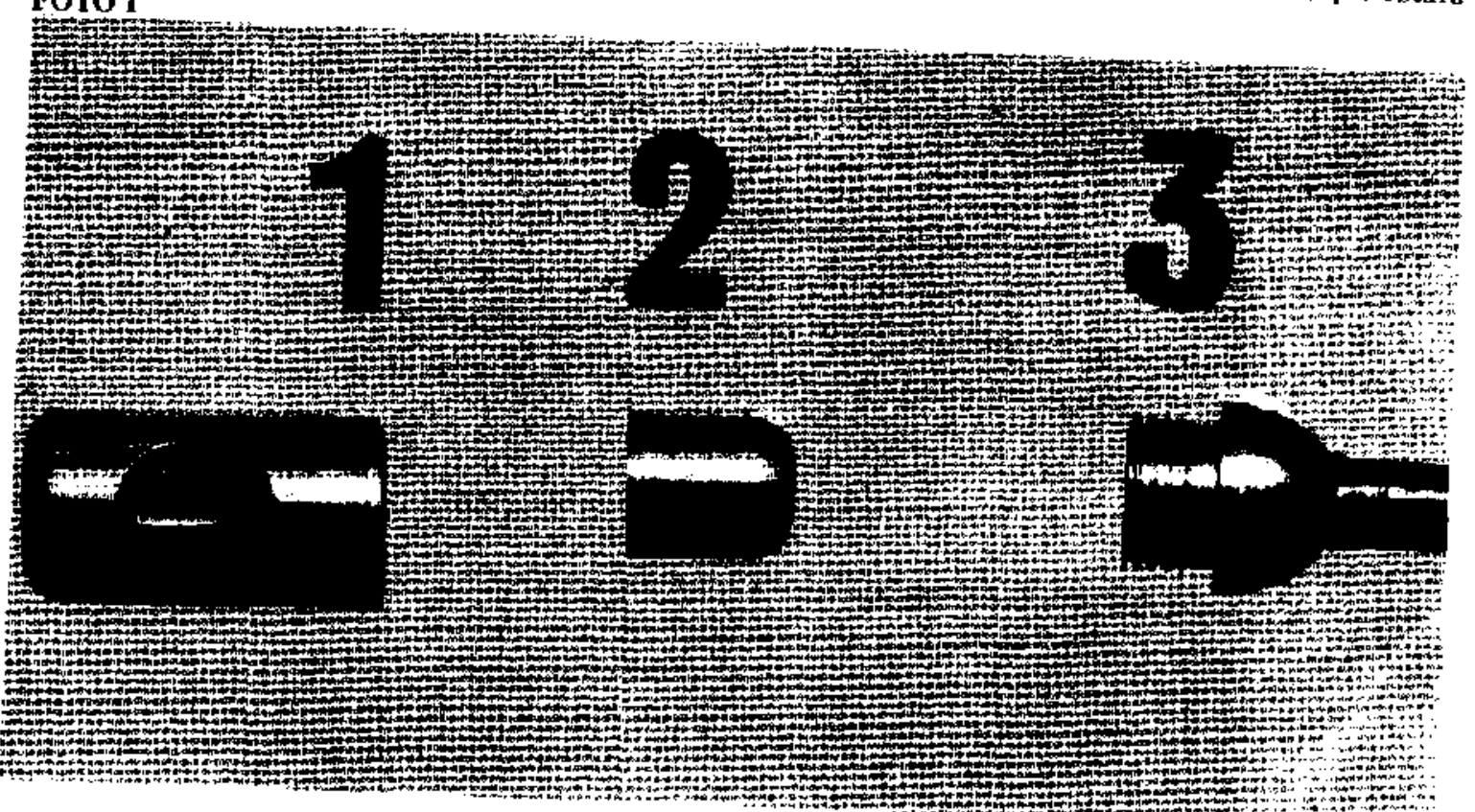

\section{OBJETIVOS}

El objetivo del autor es describir una técnica de biopsia intestinal al alcance de cualquier gastroenterólogo de provincia, que una vez puesta en prátíca. proporcionará un diagnóstico certero en sus pacientes, y por otra parte permitirá descongestionar los centros especializados en la materia.

El autor no se referiru, por el objetivo señalado, a las múltiples cápsulas que existen para tal objeto ni a los sofisticados instrumentos que en la actuaddad permiten obtener bajo cuntrol radiológico varias muestras de mucosa en escasos minutos, puesto que éstos tienen un alto costo y dependen del apoyo

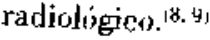

\section{MATERIAL PARA BIOPSIA INTESTINAL}

En Santiago de Chile es posible adquirir con un costo apruximado de US\$ 50 una versión criolla de Ja cápsula de Carey. de fácil manejo y excelente rendimiento.

Consta de dos piezas de metal inoxidable atornilladas entre sí, la cúpula o parte distal y la base o parte proximal. que se prolonga en un pequeño vástago, al cual se adapla y hija la sunda radioupaca. iver fotografía N." 1.)

La cüpula posee una abertura circular en la cunvexidad, y una ventanilla de 2 a $3 \mathrm{~mm}$. de diámetro a un costado, a través de la cual se obtiene la encuentra una pieza cilindrica cortante, yue obtura muestra de mucusa. En el interior de la cápsula se 
la parte distal de la cúpula y que actúa como guillotina; finnalmente contiene un pequeño resorte de acero que retiene la cuchilla, mantiene abierta la ventanilla, y cuya resistencia es necesario vencer durante la succion-biopsia.

Como sondas radioopacas pueden utilizarse las sondas para cateterismo cardiaco $N . " 8$, que se pueden obtener del material en desuso de los Centrus de Cirugía C'ardiovascular.

Una sonda va unida firmemente al vástago de la eápsula de biopsia y otra sonda, de preferencia de polietileno, se une "en paralelo" para obtener liquido intestinal.

Para evitar que las sondas sean mordidas por el paciente, durante el texamen, debe utilizarse un protector bucal de acrílico o material plástico.

Para la succión se utiliza una jeringa de $20 \mathrm{cc}$. y tinalmente, para el control del $\mathrm{pH}$, se utiliza la cinta para medir $\mathrm{pH}$ "Merck", con un rango de $\mathrm{pH}$ de 1 a 10 . \{ Ver fotogratia N.02.\}

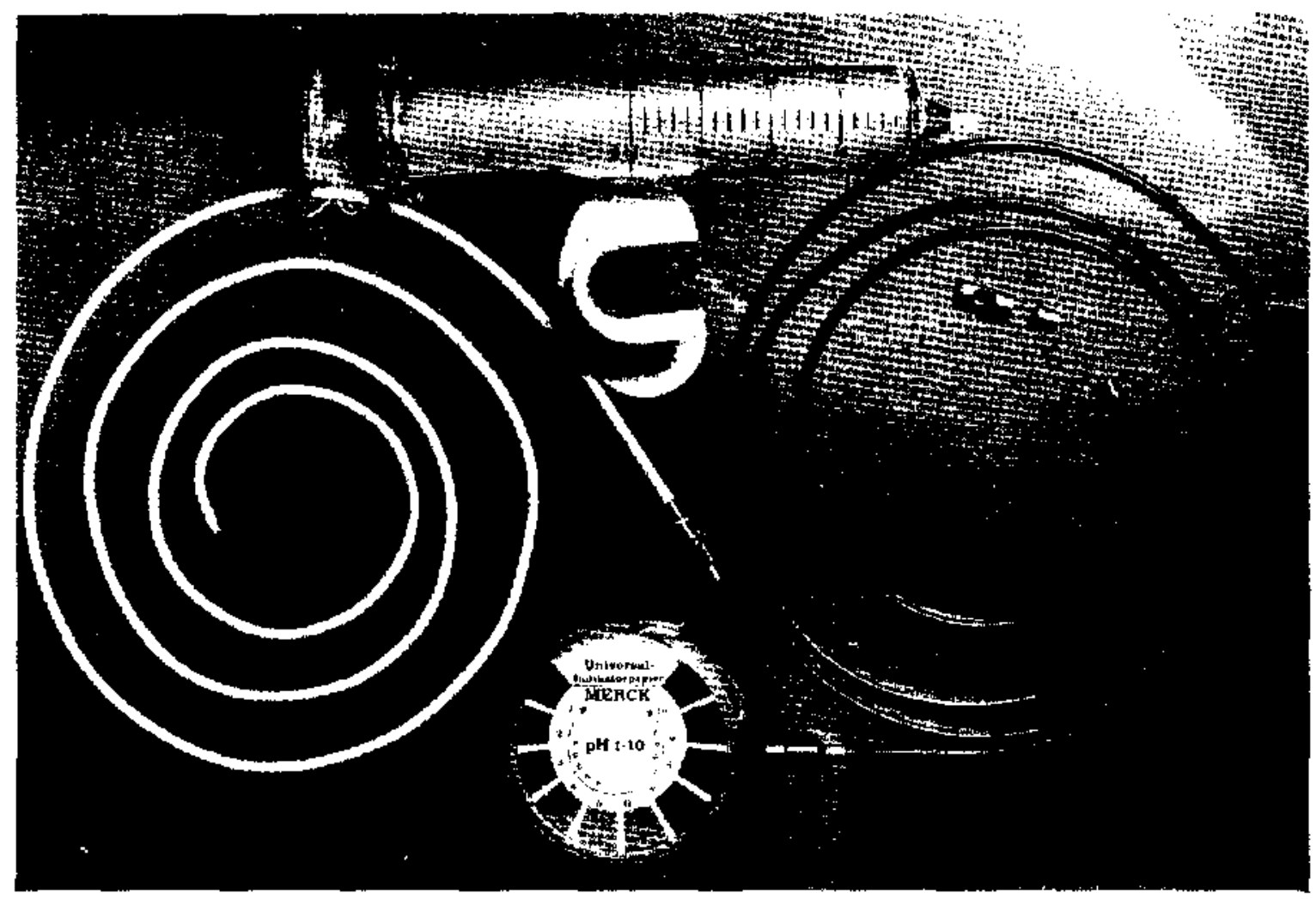

\section{PREPARACION DEL PACIEYTE Y PREMEDICACION}

Puesto que la mayoria de los pacientes sometidos a biopsia intestinal son portadores de diarrea crónica, malabsurción y esteatorrea, es aconsejable inyectar 48 horas antes del procediniento $\overline{5} \mathrm{mg}$. de vitamina $K$, por vía intramuscular. para prevenir eventuales accidentes hemorrágicos por hipoprotrombinemia.

Es indispensable medir y pesar previamente al paciente, para el cálculo de la dosis de fármacos a utilizar en la premedicación y conocer la longitud de sonda a introducir. El paciente debe estar en ayuno previo por un lapso no menor de 6 horas.

\section{Premedicación}

El autor utilizo la premedicación preconizada por H. Gaze y cols., que consiste en la sedación de Diazepam asuciado a metoclorpramide, para facilitar el paso de la cápsula a través del piloro. (10. 11. 12)

Esta asuciación medicamentosa permíte pasar la cápsula cuando la sedación es máxima y la actividad de la metoclorpramide es óptima, lo que ocurre aproximadamente a los 30 minutos de la administración. La dosis utilizada de Diazepam fue de 0.5 mg./kg. de peso corporal de "Valiimm" por via endovenosa, con un máximo absoluto de $10 \mathrm{mg}$. y metoclorpramide $0,5 \mathrm{mg} . / \mathrm{kg}$. de peso corporal, con un máximo de $10 \mathrm{mg}$. de "ITAN" por vía oral. 
La sedación es rápida y se hace efectiva en los primeros 15 minutos en el $90 \%$ de los casos. kil efecto es relativamente breve (aproximadamente dos horas), lo que permite alimentar al niño inmediatamente después del examen, condición importante en los pacientes desnutridos. Por otra parte, permite que este examen pueda efectuarse en forma ambulatoria.

Como efecto secundario de la administración de Diazepam está descrita la apnea transitoria. Sin embargo, no se observí este efecto adverso en la experiencia del autor, ni efectos secundarios atribuibles al uso de la metoclorpramide.

\section{TECNICA}

Una vez administrada la premedicacion, se inmoviliza al paciente en decúbito lateral derecho y se procede a la anestesia túpica faringea, con solución de Dimecaina al $4 \%$, y se coloca el protector bucal.

\section{Introducción de la cápsula de biopsia}

Previa comprobación del nurmal funcionamiento de la cápsula, se introduce la misma conectada a la sonda radioopaca unida "en paralelo" con tela adhesiva а ипа sonda de polietileno, que tiene por objeto controlar la ubicación de la cápsula a través de la succión, determinación del pH del fluido intestinal, y para obtenec muestras de jugo intestinal o bilis para exámenes químicos, bacteriológicos y parasitológicus.

No es recomendable utilizar la sunda unida a la cápsula para tales efectos, pues provocaría trauratismos por pellizcamientus de la mucosa intestinal, al accionarse el mecanismo de la cuchilla durante la succión.

La longitud total determinada para ja introducciún de la sonda corresponde aproximadamente al $60 \%$ de la talla (Danus y cols.). Se introduce el $50 \%$ de la longitud total determinada, lográndose con esta medida que la cápsula se ubique al nivel del tercio medio o cuerpo del estómago, para continuar la introducción, una vez obtenida la sedación máxima del paciente, aproximadamente a los treinta minutos de administrada la premedicución.

\section{Control de $\mathrm{pH}$}

El control de $\mathrm{pH}$ se realiza al iniciar el procedimiento, lo que permite comprobar el $\mathrm{pH}$ ácido del estómago, $y$, posteriormente, al iniciarse el drenaje espontáneo de bilis.

Cuando la bilis procede de retlujo, habitual- mente es turbia, y el pH fluctúa entre los rangos ácidos y neutros. La bilis pura tiene un $\mathrm{pH}$ de 8 , es de color amarillo intenso y aspecto límpido.

El autor comprobó drenaje espontáneo de bilis en el $92 \%$ de los casos, lo que permite obviar el control radiológico.

\section{Control radiologico}

Cuando el paciente experimenta náuseas y desasosiego o no se abtiene drenaje espontáneo de bilis, es necesario el cuntrol radiológico de la sunda.

Las náuseas y el desasosiego indican el enrollamiento de la sunda y habitualmente basta sentar al paciente, retirar la sonda hasta el $50 \%$ de la longitud total determinada y reiniciar la introducción para obtener la vía correcta.

\section{Obtención de la muestra}

Una vez comprobado en forma reiterada que la bilis drenada por la sonda es francamente alcalina (ph 8 ), se sncciona vigorosamente con la jeringa de $20 \mathrm{cc}$. y se procede a retirar suavemente la sonda, manteniendo la succión.

\section{Fijación de la muestra}

Luego se desarma la cápsula en un lugar bien iluminado. Ceneralmente la muestra de mucosa se encuentra atrapada en el reaurte, con la superficie epitelial dirigida al centro del mismo, basta, pues. aplicar un trozo de papel filtro a la superficie de la mucosa para que ésta se adhiera en forma correcta, con la superticie epitelial libre. Luego ge procede a extender la muestra con una aguja hipodérmica y se introduce con el papel al frascu con solución fijadora țormol, o liqujdu de Buuin, a indicación del pató$\log (0)$.

Se rotula la muestra y se envia al Laboratorio de Anatomia Patoligica.

\section{Postoperatorio}

El paciente puede ser alimentado de inmediato. Se recomienda reposo y observación por un lapso de 24 horas, en el domicilio del paciente, advirtiendo a los tamiliares que concurran al Servicio de Urgencia en caso de dolor abdominal, palidez, melena, a cualquier otro sintuma que permita sospechar una complicación.

\section{RESULTADOS}

El autor utilizó la técnica descrita en 140 pacientes, constituido en su mayoria por niños desnutridos o de baja talla, con antecedentes de diarrea crönica o 
malabsorción intestinal comprobada con el test de caroteno cun sobrecarga. ${ }^{13.171}$

La distribución por sexo correspondió a $56 \%$ femenino y $44 \%$ de sexo masculino.

Las edades fluctuaban desde 1 a 13 años. El $15 \%$ eran niños menores de 2 años; e, $65 \%$, de 2 años 1 mes, hasta 6 años, y el $20 \%$ restante correspondió a mayores de 6 años.

El estado nutritivo era normal en el $7 \%$ y desnutridos el $93 \%$. De acuerdo al criterio de Sempé, eran desnutridos leves el $52 \%$, moderados el $29 \%$ y graves el $12 \%$. El de menor peso correspondiú a un lactante de 6.700 gramus.

La premedicación se consideró altamente satisfactoria, puesto que fue efectiva en más del $90 \%$ de los casos. No se observaron efectos adversos atribuibles a la premedicación.

Se comprobó drenaje espontáneo de bilis en el $92 \%$ de los casos.

Se obtuvo muestra suficiente de mucosa para estudio histológico en el $92 \%$, cifra que es comparable a los mejores rendimientos de los casuísticos nacionales y extranjeros. ${ }^{1,}, 5,15,16,17$,

Sóto en un caso se obtuvo muestra de mucosa pilórica, y en $4 \%$ muestra de los segmentos proximales del duodeno. En el resto se obtuvo mucosa con caracteres histológicos de duodeno distal o yeyuno proximal.

El 6\% de los casos requirió control radiológico (todos lactantes).

No se observaron complicaciones descritas en otras publicaciones, tales como sangramiento intestinal significativo, perforaciones o peritonitis..$^{(1)]}$

El procedimiento total de la biopsia intestinal peroral requiere un promedio de dos horas.

El adiestramiento de una Auxiliar de Enfermería permite reducir la atención directa del médico a la introducción inicial de la sonda y a la obtención de la biopsia, una vez que se compruebe el drenaje espontáneo de bilis.

\section{RESUMEN}

Se describe una técnica de biopsia intestinal peroral con una minima implementación instrumental, que se aplicó en 140 niñus cuyas edades fluctuaban entre 1 y 13 años.
Como premedicación se utilizó Diazepam endovenose asociado a Metoclorpramida oral.

La sedación se consideró satisfactoria en el $90 \%$ de los casos.

Se practicó la succión-biopsia una vez obtenido el drenaje espontánea de bilis de $\mathrm{pH} 8$, lo que ocurrió en el $92 \%$ de los casos, permitiendo obviar el control radiológico.

Se obtuvo muestra suficiente de mucosa intestinal para estudjo histológico en el $92 \%$ de los casus, rendimiento comparable al de otras metódicas más complejas.

No se registraron complicaciones secundarias a la premedicación ni a la biopsia propiamente tal.

\section{REFERENCIAS}

I Forriatax, J. P, "La biopsie intestinale par voie acale chez l'entant. Technique et résultats". Pédiatrie, 23: 169. 1968.

2 McNicholl, B. "Yeyunal biopsy in Celiac discase". Cilinicul Pediatrics, $7: 544.1968$.

3 Jos, J, $y^{\circ}$ culs. "La bjupsie intestinale chez l"eofanr". Arch. Française de Pédiatrie, 24: 1159, 1967 .

$\downarrow$ Dunis, 0., y cots, "Entermedad Celiaca. Laracteristicas clínicas e histolígicas". Pediatria, 10: $9.1 \% 2 \mathrm{~J}$.

- Gijiruldes, E., y cols. "Biupsia inteslinal en la Enfermedal Lelíana". Rer. Médica de Lhije, 105: 553, 1976.

- Larrain, F, y cols. "Cuntraprueba terapéulica en el diagnós. tico de la Enjermedad Celíaca". Rev. Chilena de Yediatria, 45; 417, 1974.

7 Schubert, W. K "-ieliac discase. Trealment, But wilh inadequate diagnosis?" Pediatrics Vul. 61, N." 6, 1978.

${ }^{8}$ Lincheer, W. G. "A new directable small bowell Hiops; device". Gatruenterulugy, $71: 575,1976$,

* Evans, N. "-hew techriques for speeding small inlestinal biupsy". Gut 11: 88, 1970.

10 Ciaze, H., y cols. "Premedication for yeyunal biopsy in Childhowd using intravenus Diazepam and inetoclurpramide". Arch. of Disease in Childboud, 49:322, 1974.

II Harris, M. d. "Modification to the cechnique lar small bowel Biupsy in children. U6e sf meluclorpramide". Aner. Juurnal of dise ase of children, 115: 43, 1968.

12 Wakefield, M. A. "Technique fur obtainieg yeyunal biupsies in children". Arch. Wis, Child, 51: 401, 1976.

13 Cü̈iraldes, E., y culs. "El examen del caruteno séricu en la diarrea cronica de ha intancia”. Kev. Chilena de Pedautria. 45: 4/9. 1974.

14 Damis, $O$., y cols. "Tesi de caruleno plagmáticu en el diagnústico de la Entemedad Cielíaca“. Podiatría, 17: 13, 1974.

15 Kunder, E. "Perural inleatinal Biopsy in Children". Amer. Juturn. Dis. Child. $107:$ a $322,1964$.

It Siteet ty, T. W. "Intestinal Biupsy". The Lancet, 459, 196-1.

I7 Salazar de Sotusa, I. "Binpgie intestizale clez lentianl. Tiechnique et résullats.”. Pédialrie, 233: 753, 1408.

I8 Partith, J.C. "- Precautionary note on the use of the intestiral biupsy capsule in indants and emaciated Children". The Nien. England Juumal of hedicine. Vol. 2: 94, 1966. 